\title{
Повышение энергоэффективности холодильных систем в интеллектуальных сетях электроснабжения
}

\author{
М. А. Петренко ${ }^{1}$ Ф. А. Трииин ${ }^{2}$, В. А. Мазур ${ }^{2}$ \\ ${ }^{1}$ Украинский научно исследовательский институт бытового машиностроения (УкрНИИБытМаш), \\ ул. Школьная 117, г. Краматорск, Украина \\ ${ }^{2}$ Одесская национальная академия пищевых технологий, ул. Канатная, 112, г. Одесса, 65039, Украина
}

\begin{abstract}
Рассмотрена концепциия интеллектуальных сетей (Smart Grid) электроснабжения для повыщения энергоэффективности холодильных систем. Предложена модель виртуальной энергетической системы, в состав которой входят подсистемь охлаждения и отопления. Эта система включает в себя информационные $и$ коммуникационные сети наряду с технологиями сбора данных по производству $u$ потреблению энергии. Развитие таких систем автоматически приводит к увеличению устойчивости производства и распределения электроэнергии в энергетической системе в иелом, а также способствует повышению эффективности и экономической выгоды отдельных подсистем.
\end{abstract}

Ключевые слова: Холодильные системы; Энергоэффективность; Интеллектуальные сети электроснабжения

\section{Підвищення енергоефективності холодильних систем в інтелектуальних мережах електропостачання}

\author{
М. О. Петренко ${ }^{1}$ Ф. А. Трішин ${ }^{2}$ В. О. Мазур ${ }^{2}$ \\ ${ }^{1}$ Український науково-дослідний інститут побутового машинобудування (УкрНІІБитМаш), \\ вул. Шкільна, 117, м Краматорськ, Україна \\ ${ }^{2}$ Одеська національна академія харчових технологій, вул. Канатна, 112, м. Одеса, 65039, Україна
}

\begin{abstract}
Розглянуто конщеепцію інтелектуальних мереж (Smart Grid) електропостачання для підвищення енергоефективності холодильних систем. Запропоновано модель віртуальної енергетичної системи, до складу якої входить підсистеми охолодження та опалення. Ця система включає в себе інформаційні та комунікаційні мережі поряд з технологіями збору даних з виробництва і споживання енергіі. Розвиток таких систем автоматично призводить до збільшення стійкості виробниитва і розподілу електроенергї̈ в енергетичній системі в иілому, а також сприяє підвищенню ефективності та економічної вигоди окремих підсистем.
\end{abstract}

Ключові слова: Холодильні системи, Енергоефективність, Інтелектуальні мережі електропостачання

DOI: http://dx.doi.org/10.15673/ret.v53i4.708

(C) The Author(s) 2017. This article is an open access publication

This work is licensed under the Creative Commons Attribution 4.0 International License (CC BY) http://creativecommons.org/licenses/by/4.0/

\section{1 Введение}

Холодильные системы являются потребителями значительного количества энергии во всем мире. В то же время они обладают потенциалом для аккумуляции энергии в форме теплоты и холода в системах электроснабжения, в целом. Эта особенность холодильных систем позволяет управлять нагрузочными режимами в энергетической системе.

Первые попытки оценить характеристики энергосбережения холодильных систем были сделаны в середине 90-х годов прошлого столетия на основе энергетических меток. Положительная черта данного подхода - быстрая оценка конкурентоспособности различных производителей холодильного оборудования и возможность принятия решения для потребителей. В настоящее время тестирование приборов происходит при температуре окружающей среды $25^{\circ} \mathrm{C}$ и не учитывает реальные условия эксплуатации (например, открытие дверей холодильника, степень заполнения свежими продуктами холодильных камер и т. п.). Для различных холодильников условия тестирования не совпадают, что является объектом критического отношения к выводам относительно методологии энергетических меток. Аналогичные замечания относятся к подходу TEWI, в котором наряду с энергетическими показателями учитывается эмиссия углекислого газа [1].

Наиболее перспективный подход с точки зрения концепции устойчивого развития заключается в инвестировании значительных средств на «интеллектуализацию» энергетических систем. Окупаемость традиционных сетей электроснабжения обычно составляет десятки лет, в то время как 
подключение «интеллектуальной» подсистемы, хотя и увеличивает экономическую составляющую энергетической системы, может привести к уменьшению среднего периода возврата вложений до 3 -5 лет.

В настоящей работе рассматривается подход, использующий интеллектуальные сети электроснабжения (Smart grid), в состав которых наряду c информационными и коммуникационными технологиями входят технологии для сбора информации о производстве и потреблении электрической энергии. Интеллектуальные энергетические системы объединяют производителей, которые могут управлять поведением всех подключенных пользователей, с целью обеспечения устойчивого, экономичного и надежного электроснабжения.

Холодильные установки наряду с тепловыми насосами входят в состав энергетической системы и выступают в роли аккумуляторов энергии, регулирующих энергетический баланс сетей.

Разработка таких систем повышает эффективность, надёжность, экономическую выгоду, а также устойчивость производства и распределения электроэнергии [2]. Правила разработки «интеллектуальных сетей» определены в Европе через «Платформу европейских умных сетей электроснабжения» (Smart Grid European Technology Platform) [3]. Развитие технологии Smart Grid означает фундаментальную реорганизацию рынка услуг электроэнергетики, хотя терминология предполагает только технические аспекты развития сетевой инфраструктуры. Подробный обзор литературы, посвященной анализу интеллектуальных сетей, приведен в [4], где основное внимание уделено интеллектуальным инфраструктурным системам, интеллектуальным системам управления и интеллектуальным системам защиты.

Цель данной работы заключается в разработке методов снижения энергопотребления в холодильных системах в реальных условиях эксплуатации за счет использования технологии Smart Grid.

Вначале мы рассматриваем агентные модели виртуальных энергетических систем. Далее, в качестве аккумулятора теплоты представлена агентная модель виртуальной холодильной системы. Четвёртый раздел посвящен описанию возможных критериев управления виртуальной энергетической системой с точки зрения достижения компромиссного решения в рамках концепции устойчивого развития. В пятом разделе приведены результаты, иллюстрирующие повышение энергоэффективности холодильных систем за счет интеллектуального управления ресурсами всей энергетической системы. Наконец, в заключение обсуждаются перспективы применения умных сетей в холодильной технике.

\section{2 Агентные модели виртуальных энергетических систем}

Энергетическая система представляет собой сложную систему, управление которой в реальных условиях трудно или невозможно осуществить.
Моделирование таких сложных энергетических систем целесообразно реализовать при помощи виртуальных компьютерных мультиагентных моделей $\{\mathrm{MAS}\}$, которые имитируют взаимодействие автономных подсистем (агентов) между собой и окружающей средой с целью интегральной оценки эффективности системы. Термин «агент» реализует программную идею или концепцию и определяется посредством описания его поведения [5]. Представление об агенте обеспечивает удобный способ описания сложной системы, которая способна автономно действовать с целью выполнения задач от имени пользователя. Виртуальная энергетическая система (рисунок 1) является связующим звеном между различными типами генераторов теплоты и электроэнергии и позволяет устранить противоречие между требованиями рынка и возможностями технической реализации.

\section{Виртуальная энергетическая система (ВЭС)}

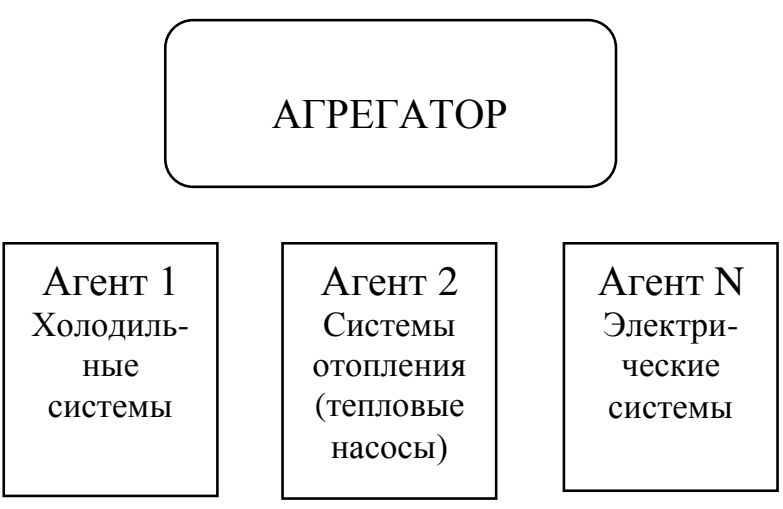

Рисунок 1 - Мультиагентная система

Существуют различные определения агента, включающие такие понятия, как [5]:

- живучесть (код не исполняется по требованию, а работает непрерывно и решает сам, когда он должен совершить какие-либо действия);

- автономность (агент имеет способность выбора задач, приоритетов, целенаправленного поведения и принятия решений без участия человека);

- социальное поведение (агенты имеют возможность привлекать другие компоненты посредством определённых связей и координации действий, они могут совместно работать над выполнением задач);

- реактивность (агенты воспринимают контекст, в котором они работают, и реагируют на него должным образом).

Системы MAS относятся к числу так называемых «самоорганизующихся систем, которые, как правило, находят наилучшее решение своих проблем без вмешательства извне.

\section{3 Агентная модель виртуальной холодильной системы}

Методы компьютерной флюидной динамики успешно применяются при решении нестационарных задач. Однако затраты на вычисления при этом 
значительно возрастают. Более целесообразно рассмотреть упрощенные динамические модели переходных процессов в камерах холодильника, что позволит оперативно получить ответ о влиянии различных факторов на переход холодильной системы из неравновесного в стационарное состояние. На рисунке 2 рассмотрена агентная модель парокомпрессионной

холодильной

системы, включающая основные элементы: конденсатор (Кд), компрессор (К), испаритель (И) и дроссель (Др).

Основные уравнения динамики объекта охлаждения, отражают баланс энергии в традиционной холодильной системе.

\section{Объект охлаждения} (3)

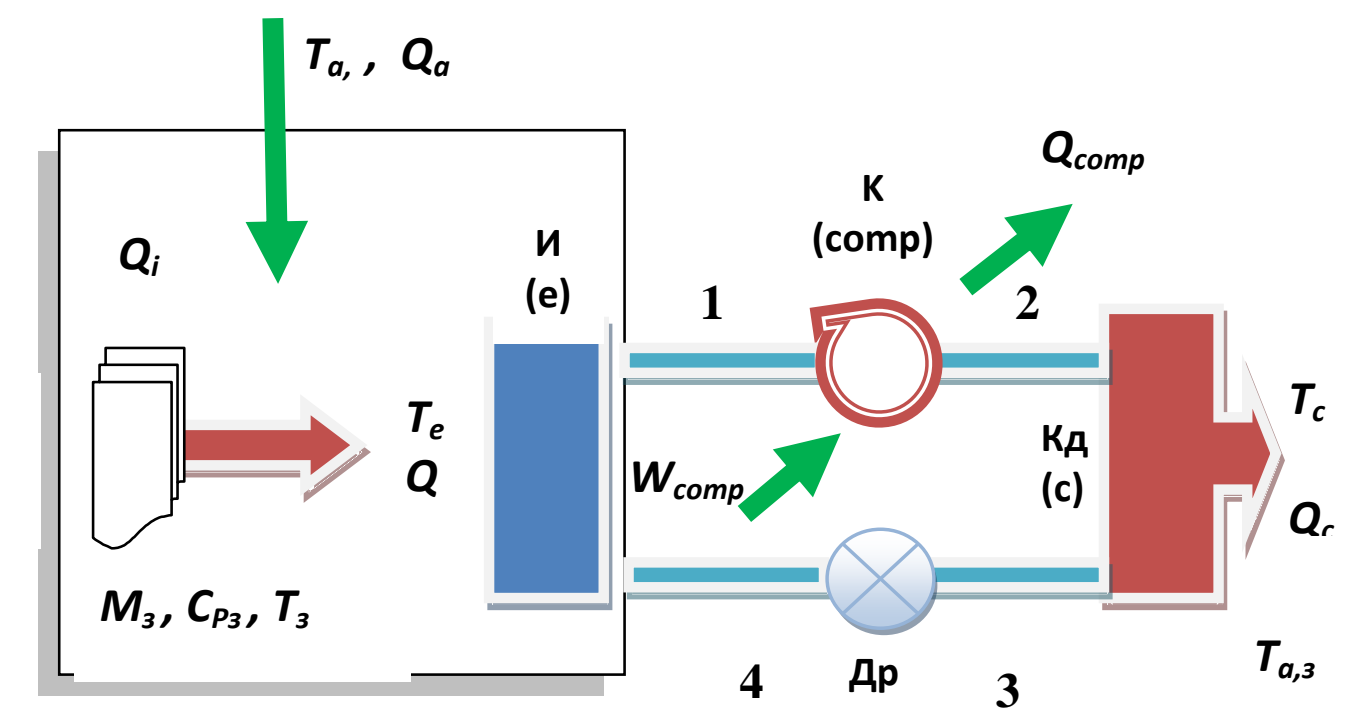

Рисунок 2 - Основные элементы агентной модели холодильной системь

Объект охлаждения (загрузка)

$$
\begin{aligned}
& M_{3} C_{P 3} \frac{d T_{3}}{d \tau}=Q_{a}+Q_{i}-Q_{e} . \\
& Q_{a}=U A_{a}\left(T_{a}-T_{3}\right)
\end{aligned}
$$

Испаритель

$$
\begin{aligned}
& Q_{e}=\dot{m}_{\text {comp }}\left(h_{1}-h_{4}\right) \\
& Q_{e}=U A_{e}\left(T_{3}-T_{e}\right)
\end{aligned} .
$$

Конденсатор

$$
\begin{aligned}
& Q_{c}=\dot{m}_{\text {comp }}\left(h_{2}-h_{3}\right) \\
& Q_{c}=U A_{c}\left(T_{c}-T_{a, \text { cond }}\right)
\end{aligned}
$$

Дроссель

$$
h_{4}=h_{3} \text {. }
$$

\section{Компрессор}

$$
\begin{aligned}
\dot{m}_{\text {comp }} & =\eta_{\text {vol }} \rho_{1} \dot{V}_{d} \\
\dot{m}_{\text {comp }} & =\left(h_{2}-h_{1}\right)=W_{\text {comp }}-Q_{c o m p} \\
W_{\text {comp }} & =\frac{\dot{m}_{c o m p}\left(h_{2 s}-h_{1}\right)}{\eta_{s}} \\
Q_{c o m p} & =Q_{f} W_{c o m p}
\end{aligned}
$$

Здесь использованы следующие обозначения: $Q$ - теплота, $W$ - работа; $M$ - масса; $C_{P}$ - изобарная теплоемкость; $h$ - удельная энтальпия; $U A$ произведение коэффициента теплопередачи на площадь; $\dot{m}$ - расход хладагента; индекс «а» характеризует окружающую среду. Остальные индексы указаны на рисунке 2. Система дифференциальных уравнений (1)...(5) легко решается численно при помощи стандартных программ.

Вычисление энтальпии и энтропии проводили по уравнению Редлиха-Квонга [6] по известным термодинамическим соотношениям:

$$
p=\frac{R T}{V-b}-\frac{a}{\sqrt{T} V(V+b)} .
$$


Для определенности рассматривали хладагент R134a. В частности, энтропию в заданной точке термодинамической поверхности $\{T, V\}$ по уравнению состояния вычисляли по формуле:

$$
S(T, V)=R \ln (V+b)+\frac{a \ln \frac{V}{V+b}}{2 b T \sqrt{T}}+c_{0} \ln T+c_{1} T+\text { const }
$$

при известной идеально-газовой изохорной теплоемкости $C_{V}^{i d}=c_{0}+c_{1} T$. Выбор произвольной постоянной проводили на основе стандарта Международного института холода $h_{0}=200$ Дж/кг: $S_{0}=1$ кДж/кг/К для жидкости в состоянии насыщения при $0^{\circ} \mathrm{C}$. Необходимые данные о теплоемкости в идеально-газовом состоянии взяты из Веб справочника NIST (http://webbook.nist.gov).

В качестве входных параметров при работе программы использовали данные о температуре окружающей среды для широты г. Одессы в марте 2016 года (рисунок 3).

Начальную температуру объекта охлаждения принимали равной $25^{\circ} \mathrm{C}$.

Теплоемкость объектов охлаждения выбирали в интервале: $2.85 \ldots 3.38$ кДж/кг/град, что соответствует теплоемкости мяса по данным http://aiche.confex.com/aiche/2005/techprogram/P17990.H TM.

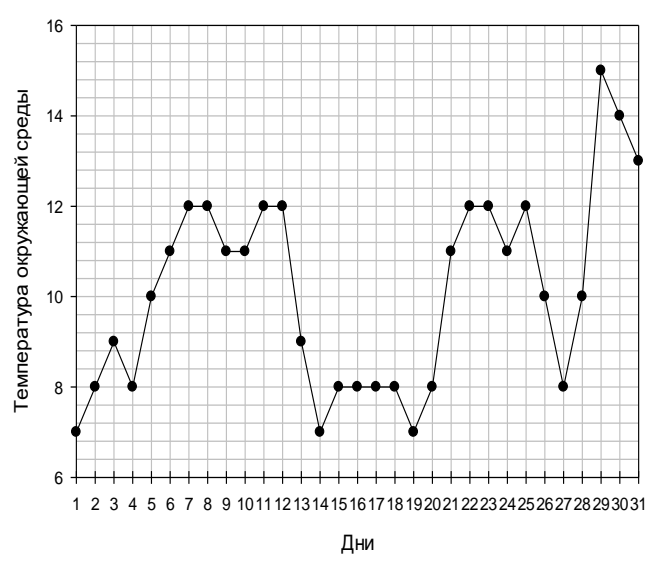

Рисунок 3 - Температура окружающей среды

Усредненные характеристики холодильной системы принимали равными: изоэнтропийная эффективность компрессора - 0,8 ; объемная эффективность - 0,85; объемная производительность $1,8 \mathrm{~m}^{3} /$ час; перегрев в испарителе - $5^{\circ} \mathrm{C}$; переохлаждение в конденсаторе $-5^{\circ} \mathrm{C}$; время перехода в стационарное состояние - 3600 сек.

Информация на выходе из программы включает: температуры объекта охлаждения, конденсатора, испарителя, холодильный коэффициент - COP, энергопотребление (работа компрессора - $W$ ) и теплоту, передаваемую хладагентом в конденсаторе и испарителе.

\section{4 Критерии управления и нечеткая мульти- агентная оптимизация виртуальной энерге- тической системы}

В принципе, возможны различные критерии управления энергетической системой в зависимости от требований потребителя. Наиболее распространенные критерии управления приведены ниже.

Прямое управление. Здесь предполагается, что цели потребителей и производителей совпадают и минимизируют эксплуатационные расходы для всей энергетической системы. В этом случае взаимосвязь между потребителями и генерирующей компанией позволяет осуществить прямое управление спросом. Аппаратная реализация системы управления может быть достаточно простой, поскольку только отправляет информацию и получает команды от управляющих параметров энергетической системы. Недостатком такого критерия является необходимость решать крупномасштабные задачи оптимизации для координации большого количества агентов. Поскольку управляющие параметры всей энергетической системы полностью определяют график потребления агентов, то окончательный результат не является оптимальным для потребителя.

Контроль частоты. Потребитель измеряет частоту в сети, которая в Европе имеет номинальное значение 50 Гц. Когда спрос превышает предложение частота падает, и наоборот, когда предложение превышает спрос частота увеличивается. Преимуществом такого типа управления является низкая цена контроллера, поскольку фактическая активация становится автоматической и линейно зависит от частоты.

Концепция ВЭС создает огромное количество возможностей для балансировки нагрузки, поскольку позволяет активно управлять потребителем [7, 8, 9]. Оценка перспективности и оптимизация процессов требуют развития вычислительных инструментов, которые адекватно отображают надежные экспериментальные данные [10].

Для поиска оптимального решения развита гибкая модель мультиагентного анализа, базирующаяся на многокритериальной природе процесса принятия решения в Smart Grid при наличии конфликта и нечеткости целей при проектировании энергопреобразующих систем.

С математической точки зрения задача реализации концепции устойчивого развития рассматривается как задача мультиагентной оптимизации, которая сводится к проблеме нечеткого нелинейного программирования с $n$ несовместимыми критериями (экономический, социальный, термодинамический и другие), $m$ переменными управления и $k$ нелинейными ограничениями: найти

$$
\text { Optimize } K\left[K_{l}(X), \ldots, K n(X)\right]
$$

при условии

$$
\begin{gathered}
C_{i} \equiv G_{L i} \leq G_{i}(X) \leq G_{U i}, i=1,2, \ldots, k \\
x_{L i} \leq x_{i} \leq x_{U i}, i=1,2, \ldots, m,
\end{gathered}
$$


где $K_{i}(X)(i=1 \ldots n)$ представляют нечеткие локальные критерии эффективности ВЭС; $X\left(x_{1}, x_{2}, \ldots, x_{m}\right)$ - вектор искомых переменных управления; $G_{L i}, G_{U i}-$ нижний и верхний пределы ограничений $G_{i}(X$,$) , соответственно,$ и $x_{L i} \leq x_{i} \leq x_{U i}-$ нижняя и верхняя границы для искомых переменных управления.

Мы полагаем, что $K_{j}(X)=\left\|P_{j}, M_{j}(X)\right\|-$ «расстояние» между желаемой (идеальной) эффективностью системы $P_{j}$ и ее реальной моделью $M_{j}$. Решение многокритериальной проблемы заключается в нахождении компромисса среди всех критериев и ограничений и формулируется следующим образом: построить функцию

$$
K=K_{1} \bigcap K_{2} \bigcap \ldots \bigcap K n
$$

Параметры модели $X_{o p t}$ отождествляют компромиссное решение для выбранных критериев эффективности. Многокритериальный подход базируется на комбинации формальной и неформальной процедур принятия решений для нахождения приемлемых альтернатив.

В данной работе предлагается следующая последовательность шагов принятия решения в мультиагентной оптимизации ВЭС.

- Определение области оптимальности по Парето (или области компромисса) $-X_{P}$, в которой достигается согласованное решение конфликта между критериями с противоположными интересами;

- Представление критериев и ограничений в форме нечетких множеств для отображения неструктурированных ситуаций (т. н. процедура «размывания» критериев);

- Неформальный выбор схемы свертки для перехода от векторного критерия $K\left[K_{i}(X)\right.$, к скалярной комбинации $K_{i}(X)$;

- Оценка окончательного вектора $X_{\text {opt }} \in X_{P}$, минимизирующего нечеткие источники неопределенности и отражающего достижение согласованного критерия устойчивого развития ВЭС.

5 Повышение энергоэффективности холодильных систем за счет интеллектуального управления ресурсами всей энергетической системы

Для наиболее простой оценки повышения энергоэффективности холодильной системы в рамках ВЭС в работе использовали пакет моделирования AnyLogic $^{\text {TM }}[11]$, в котором реализован агентный подход к моделированию в различных сферах деятельности. Агентные модели позволяют получить представление об общем поведении системы, исходя из предположений о поведении ее элементов, при отсутствии знания о глобальных законах - то есть в наиболее общем случае. AnyLogic ${ }^{\text {тм }}$ является одним из успешных инструментов моделирования, позволяющим оперативно создавать гибкие модели с агентами, взаимодействующими как друг с другом, так и со своим окружением. AnyLogic пм поддерживает все возможные способы задания поведения агентов - диаграммы состояний (стейтчарты), синхронное и асинхронное планирование событий.

Для задания поведения агентов были введены две переменные: adopters (реальные потребители электроэнергии) и potential_adopters (потенциальные потребители электроэнергии), для которых задавали свойства на диаграмме состояний. Кроме того, использовали опции «Действие» при входе в состояние и «Действие» при выходе из этого состояния. При входе в состояние значение переменной увеличивали на единицу, если количество потенциальных агентов подключается к энергетической системе. При выходе из состояния значение этой переменной будет уменьшаться.

В качестве иллюстрации рассматриваемого подхода задавали количество агентов $(n=100)$, что интерпретируется как модель энергетической системы супермаркета или многоквартирного «умного дома». При этом автоматически создается 100 экземпляров класcа Person, каждый из которых персонифицируется как отдельный агент. Характеристики агента задаются с помощью параметров класса и в нашем случае все агенты обладают общей структурой, поскольку принадлежат объектам одного класса. В принципе, параметры индивидуально характеризуют каждый агент. Критерий устойчивого развития выбирали в простейшей форме свертки термодинамического и экономического показателей. Управляемый выход характеризовали измеренным температурным состоянием.

Главная задача модели жизненного цикла ВЭС изучение того, как быстро агенты обращаются к источникам электроэнергии. Поэтому добавили возможность отслеживания того, сколько агентов уже обратилось к источникам энергии, а сколько - еще нет. Подсчет числа реальных и потенциальных потребителей электроэнергии проводили с помощью изучения динамики моделируемого процесса в программе AnyLogic. На рисунке 4 представлена динамика подключений (отключений) потенциальных и реальных потребителей энергии для ВЭС со 100 агентами.

Динамика подключений агентов

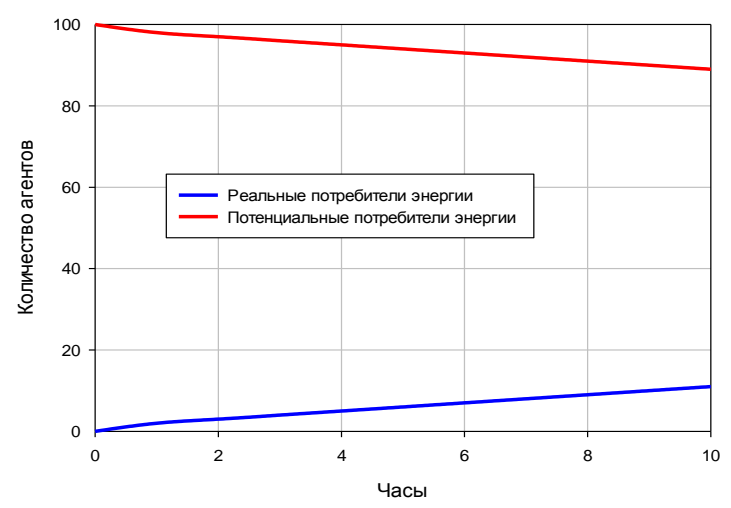

Рисунок 4 - Динамика процессов потребления энергии агентами 
Существенной проблемой являются потери при пуске и остановке компрессора. На рисунке 5 изображены колебания СОР при неблагоприятных внешних условиях, вызывающих частое включение / выключение компрессора. В данном случае внешние факторы имитировали ухудшением процессов теплопередачи между окружающей средой и холодильной системой. Минимизация переключений компрессора приводит к снижению энергопотребления до $30 \%$ (оптимистическая оценка). Пессимистическая оценка составляет порядка 7. Компромиссный вариант переключения компрессора на основе критерия устойчивого развития достигает $10 \%$. Динамика изменения холодильного коэффициента для этого случая представлена на рисунке 6.

Основные факторы, влияющие на энергосбережение - это размер и эффективность компрессора, системы температурного контроля и размер испарителя. Как показывают расчеты, не все факторы являются равноценными и не всегда указывают основные направления по уменьшению потребления энергии. Применение указанной модели обнаруживает несколько интересных результатов.

Например, увеличение массы загрузки не оказывает принципиального влияния на энергопотребление, поскольку десятикратное увеличение массы груза приводит к увеличению энергопотребления всего на 2\%. Теплоемкость загружаемого продукта также практически не оказывает влияние на энергозатраты. Пятикратное увеличение теплоемкости продукта приводит к небольшому (до 1,5\%) увеличению энергопотребления.

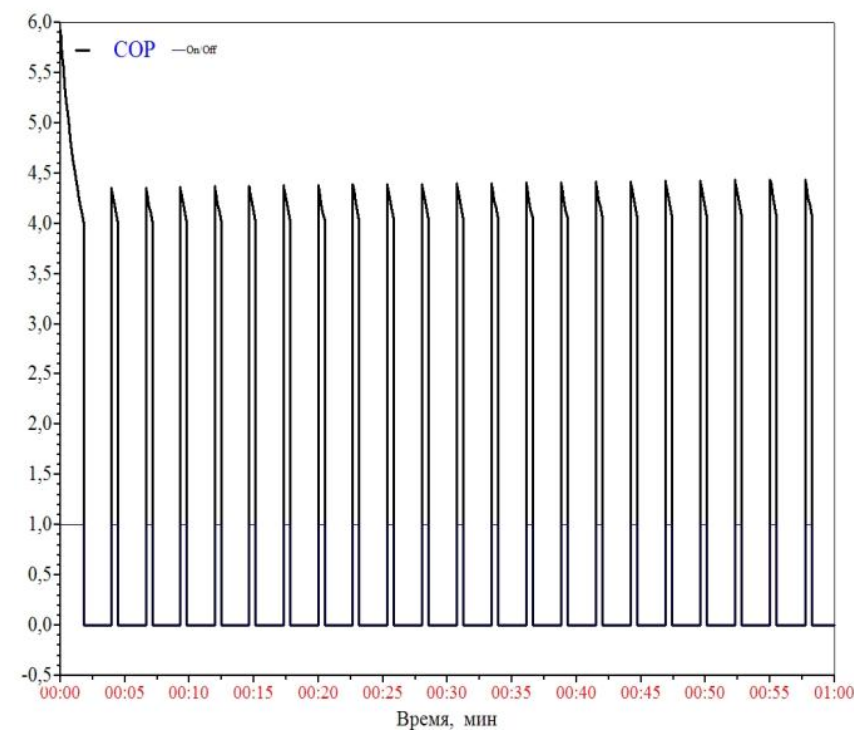

Рисунок 5 - Динамика изменения холодильного коэффиџиента при переключении компрессора (неблагоприятный режим)

На рисунке 7 представлены результаты расчетов динамики изменения температуры объекта охлаждения, температур в конденсаторе и испарителе для холодильной системы, использующей в качестве рабочего вещества R134a.

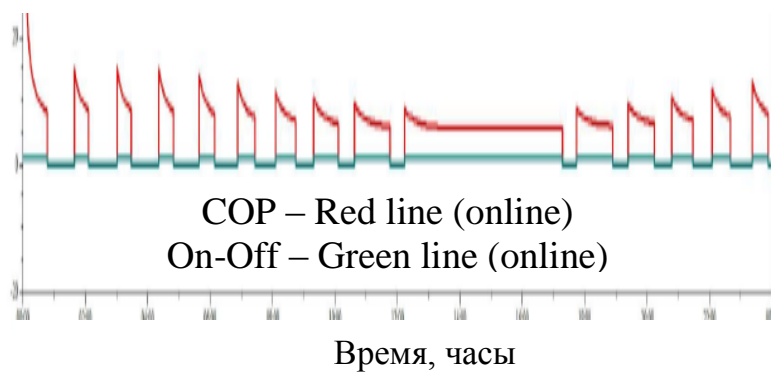

\section{Рисунок 6 - Динамика изменения холодильного коэффициента (СОР) при переключении компрессора (компромиссный режим)}

Более заметная экономия электроэнергии достигается при увеличении размеров испарителя. и компрессора. Чем больше энергопотребление, тем больше размеры компрессора. Оптимальное значение энергопотребления достигается, когда используется компрессор с минимальными размерами, обеспечивающий требуемую температуру охлаждения. Однако на практике при увеличении изоэнтропийной эффективности компрессора от 0,6 до 0,8 необходимо увеличение объемной производительности в 4 раза.

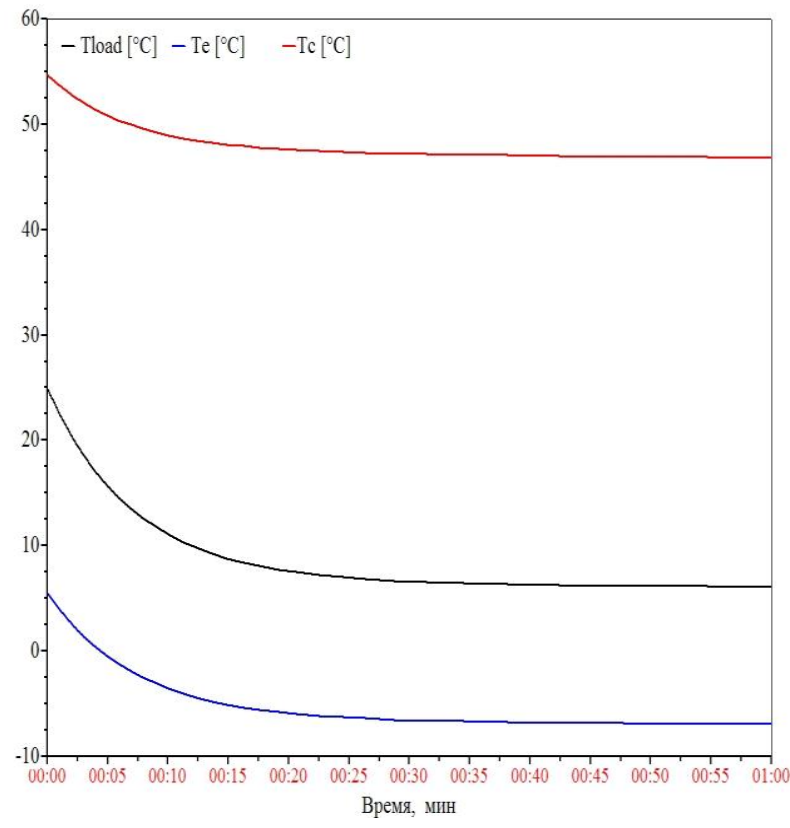

Рисунок 7 - Динамика изменения температуры в конденсаторе и испарителе для компромиссного варианта

В рамках данной работы мы не рассматривали возможное локальное повышение эффективности отдельного агента, что представляет самостоятельный интерес.

\section{6 Выводы}

Чтобы справиться с высокой сложностью будущих систем электроснабжения, где распределение энергии 
происходит между автономно действующими и взаимодействующими агентами, предпочтение следует отдать современной технологии на базе многоагентных систем (MAS). Они уже продемонстрировали свой потенциал при разработке надежных, гибких и адаптивных систем промышленной автоматизации в таких областями, как контроль производства, динамическая маршрутизация продукта, планирование производства и логистика, аэрокосмическая промышленность, управление воздушным движением и многие другие. Сочетание представлений об интеллектуальных сетях энергоснабжения и концепция нечетких множеств позволяют принимающему решения вести всеобъемлющее исследование энергопреобразующих систем, принимая во внимание разнообразные комбинации экологических и экономических целей наряду с энергетическими ограничениями. Метод нечеткого нелинейного математического программирования, который пытается минимизировать все виды неопределенностей, - это гибкая система экономической и термодинамической оптимизации энергетических систем.

В этом исследовании сделана одна из первых попыток применения методологии многокритериального принятия решений для поиска компромисса между различными показателями в интеллектуальных сетях электроснабжения, что, в конечном итоге, снижает потребление энергии холодильных систем.

Представление об умных сетях играет ключевую роль в будущих энергетических сценариях, которые стремятся обеспечить наилучшее согласование между производством и потреблением энергии. Интеллектуальные сети выходят за рамки просто технологий и следует признать, что умный пользователь оказывает большое влияние на потребление энергии. Взаимодействие потребителей энергии в рамках интеллектуальной энергетической системы является одним из перспективных направлений в реализации масштабных социотехнических систем в энергетике будущего [12].

Работы с интеллектуальными электрическими сетями требуют новых компетенций и надлежащего обучения, которые в существующей сейчас системе образования отсутствуют. Для преодоления такой ситуации необходимо разработать программу развития национальных квалификационных требований и норм, сформировать профессиональные стандарты соответствующих профилей компетенции для отдельных специальностей, использовать систему профессиональной сертификации в образовательном процессе.

\section{Литература}

1. Refrigerating Systems and Heat Pumps - Safety and environmental requirements, 2008. European Standards.

2. https://energy.gov/oe/activities/technology-

development/grid-modernization-and-smart-grid

3. Smart Grids European Technology Platform www.smartgrids.eu. smartgrids.eu (2011)

4. Fang X., Misra S., Xue G., and Yang D. 2012. Smart Grid - The New and Improved Power Grid: A Survey. IEEE Communications Surveys \& Tutorials, Vol. 14, No. 4, Fourth Quarter 2012 .doi: 10.1109/SURV.2011.101911.00087

5. Вулдридж М., Дженнингс Н. 1995. Интеллектуальные агенты: теория и практика. Knowledge Eng. Rev., vol. 10(2), pp. 115-152.

6. Redlich O., Kwong J., 1949. On the Thermodynamics of Solutions Equation of State: Fugacities of Gaseous Solutions. Chem. Rev. 44, pp. 233 -244.

7. Biegel, B., Andersen, P., Pedersen, T.S., Nielsen, K.M., Stoustrup, J., and Hansen, L.H. 2013). Smart grid dispatch strategy for on/off demand-side devices. In Proceedings of the European Control Conference 2013 Zürich, Switzerland.

8. Stadler M., Krause W., Sonnenschein M., and Vogel U., 2009. "Modelling and evaluation of control schemes for enhancing load shift of electricity demand for cooling devices," Environmental Modelling \& Software, vol. 24, pp. 285-295

9. Pedersen, R., R., Schwensen, J., Sivabalan, S., Corazzol, C., Shafiei, S.E., Vinther, K., and Stoustrup, J. 2013. Direct control implementation of a refrigeration system in smart grid. In Proceedings of the 2013 American Control Conference. Washington,

DC, USA. U.S. Department of Energy.

10. Gong J., Xie D., Jiang C. and Zhang Y. 2011. Multiple Objective Compromised Method for Power Management in Virtual Power Plants Energies 4, 700-716. 11. http://www.xjtek.com/products/anylogic

12. Goulden M., Redwell B., Rennick-Egglestone S., Tom Rodden T., Spence A. 2014. Smart grids, smart users? The role of the user in demand side management Energy Research \& Social Science 2, 21-29

Отримана в редакції 19.04.2017, прийнята до друку 06.06.2017

\title{
Energy Efficient Refrigeration Systems in a Smart Grid
}

\author{
M. O. Petrenko ${ }^{\text {, F. A. Trishin }}{ }^{2}$, V. O. Mazur ${ }^{2}$ \\ ${ }^{1}$ Ukrainian Research Institute of Household Engineering (UkrNIIBytMash). 117, Shkolnaya str., Kramatorsk, Ukraine \\ ${ }^{2}$ Odesa National Academy of Food Technologies, 112, Kanatna str., Odesa, 65039, Ukraine
}

The concept of intelligent networks (Smart Grid) for power supply to increase the energy efficiency of refrigeration systems is considered. A model of a virtual power system is proposed, which includes subsystems for cooling and heating. The paper discusses an approach where intelligent power supply network is applied. This system includes the information and communication networks along with technologies for collecting data on energy production and 
consumption. The development of such systems automatically leads to an increase in the sustainability of production and distribution of electricity in the energy system as a whole, and contributes to increasing the efficiency and economic benefit of individual subsystems.

Keywords: Refrigeration Systems - Energy Efficiency - Smart Grid

\section{References}

1. Refrigerating Systems and Heat Pumps - Safety and environmental requirements, 2008. European Standards.

2. https://energy.gov/oe/activities/technologydevelopment/grid-modernization-and-smart-grid 3. Smart Grids European Technology Platform www.smartgrids.eu. smartgrids.eu (2011)

4. Fang X., Misra S., Xue G., and Yang D. 2012. Smart Grid - The New and Improved Power Grid: A Survey. IEEE Communications Surveys \& Tutorials, Vol. 14, No. 4, Fourth Quarter 2012 .doi: 10.1109/SURV.2011.101911.00087

5. Вулдридж М., Дженнингс Н. 1995. Интеллектуальные агенты: теория и практика. Knowledge Eng. Rev., vol. 10(2),

pp. $115-152$.

6. Redlich O., Kwong J., On the Thermodynamics of Solutions: V: An Equation of State: Fugacities of Gaseous Solutions. 1949. Chem. Rev. 44, pp. 233 -244.

7. Biegel, B., Andersen, P., Pedersen, T.S., et al. 2013. Smart grid dispatch strategy for on/off demand-side devices. In Proceedings of the European Control Conference 2013 Zürich, Switzerland
8. Stadler M., Krause W., Sonnenschein M., and Vogel U., 2009. "Modelling and evaluation of control schemes for enhancing load shift of electricity demand for cooling devices," Environmental Modelling \& Software, vol. 24, pp. 285-295.

9. Pedersen, R., Schwensen, J., Sivabalan, S., Corazzol, C., Shafiei, S.E., Vinther, K., and Stoustrup, J. 2013. Direct control implementation of a refrigeration system in smart grid. In Proceedings of the 2013 American Control Conference. Washington, DC, USA. U.S. Department of Energy.

10. Gong J., Xie D., Jiang C. and Zhang Y. 2011. Multiple Objective Compromised Method for Power Management in Virtual Power Plants Energies 4, 700-716.

11. http://www.xjtek.com/products/anylogic

12. Goulden M., Redwell B., Rennick-Egglestone S., Rodden T., Spence A. 2014. Smart grids, smart users? The role of the user in demand side management Energy Research \& Social Science 2, 21-29 